\title{
Non-Linear Ion-Acoustic Solitary Waves in Electron-Positron-Ion Plasma with Non-Thermal Electrons
}

\author{
S. K. Anguma1, I. Habumugisha ${ }^{2,3}$, L. Nazziwa ${ }^{4,5}$, E. Jurua ${ }^{5}$, N. Noreen ${ }^{6}$ \\ ${ }^{1}$ Department of Physics, Muni University, Arua, Uganda \\ ${ }^{2}$ Department of Physics, Kabale University, Kabale, Uganda \\ ${ }^{3}$ Department of Physics, Islamic University in Uganda, Mbale, Uganda \\ ${ }^{4}$ Department of Physics, Bishop Stuat University, Kakoba, Uganda \\ ${ }^{5}$ Department of Physics, Mbarara University of Science and Technology, Mbarara, Uganda \\ ${ }^{6}$ Department of Physics, Forman Christian College (Chartered University), Lahore, Pakistan \\ Email:s.anguma@muni.ac.ug
}

How to cite this paper: Anguma, S.K. Habumugisha, I., Nazziwa, L., Jurua, E. and Noreen, N. (2017) Non-Linear Ion-Acoustic Solitary Waves in Electron-Positron-Ion Plasma with Non-Thermal Electrons. Journal of Modern Physics, 8, 892-902. https://doi.org/10.4236/jmp.2017.86055

Received: March 21, 2017

Accepted: May 21, 2017

Published: May 26, 2017

Copyright (C) 2017 by authors and Scientific Research Publishing Inc. This work is licensed under the Creative Commons Attribution International License (CC BY 4.0).

http://creativecommons.org/licenses/by/4.0/

\begin{abstract}
Ion-acoustic solitary (IAS) waves in electron-positron-ion (e-p-i) plasma have been of interest to many researchers probably due to their relevance in understanding the Universe. However, the study of non-linear ion-acoustic waves in e-p-i plasma with non-thermal electrons has not been adequately studied. A theoretical investigation on non-linear IAS waves in e-p-i plasma comprising of warm inertial adiabatic fluid ions and electrons that are kappa distributed, and Boltzman distributed positron is presented here using the Sagdeev potential technique. It was found that existence domains of finite amplitude IAS waves were confined within the limits of minimum and maximum Mach numbers with varying $\kappa$ values. For lower values of $\kappa$, the amplitude of the solitary electrostatic potential structures increased as the width decreased, while for high values, the potential amplitude decreased as the width of the solitary structure increased.
\end{abstract}

\section{Keywords}

Non-Thermal Electrons, Ion-Acoustic Solitary Waves, Electron-Positron-Ion Plasmas

\section{Introduction}

The electron-positron (e-p) plasmas have frequent occurrence in the Universe [1]. The inclusion of an ion in an e-p forms an e-p-i plasma. These e-p-i plasma are ubiquitious in astronomical environments. In such situations, positrons 
drastically change the behaviour of non-linear waves and it is for this reason that they have attracted the attention of several authors [2] [3] [4] [5].

Large amplitude ion-acoustic waves in e-p-i plasma were studied by [2], using the Sagdeev potential technique. Their results showed that the larger the relative positron density, the lower the Mach number for a soliton of fixed amplitude to propagate. Later, [3], studied the effect of the ion temperature on large amplitude ion-acoustic waves in e-p-i plasma consisting of Boltzmann distributed positrons. He found that the ion temperature increased the maximum Mach number and decreased the amplitude of ion-acoustic waves. Furthermore, in studying non-linear acoustic excitations in e-p-i plasma, [4] studied the accretion disks of active galactic nuclei, where the ion temperatures were much higher than those of electrons and positrons. Due to very high ion temperatures in accretion disks, the ions were modeled using the Boltzmann distribution, whereas the electrons and positrons were governed by the fluid equations. Most research work about e-p-i plasmas have treated electrons as being Boltzmann distributed species thereby, leaving kappa distributed electrons not adequately studied.

The kappa $\kappa$ distribution function possesses the desired property that the particles with velocities greater than the thermal velocity obey a power law distribution and gives better fits to real space plasma [6]. The family of isotropic generalised Lorentzian or $\kappa$-distributions takes the form [7]:

$$
F_{\kappa}(v)=\frac{N_{e 0}}{\left(\pi \kappa \theta^{2}\right)^{\frac{3}{2}}} \cdot \frac{\Gamma(\kappa+1)}{\Gamma\left(\kappa-\frac{1}{2}\right)}\left(1+\frac{v^{2}}{\kappa \theta^{2}}\right)^{-(\kappa+1)},
$$

where $N_{e 0}$ is the unperturbed equilibrium electron density, $\theta$ is the most probable speed of the particle species related to the usual thermal velocity,

$V_{t}=\left(\frac{K_{B} T}{m}\right)^{\frac{1}{2}}$ by $\theta=\left(\frac{2 \kappa-3}{\kappa}\right)^{\frac{1}{2}} V_{t} ; T$ being the characteristic kinetic temperature, i.e., the temperature of equivalent Maxwellian distribution with same average kinetic energy; $K_{B}$ is Boltzmann's constant, $\Gamma$ is the usual gamma function, and $\kappa$ is the spectral index that determines the hardness of the energy spectrum corresponding to the presence of excess suprathermal particles in the tail of the distribution function [8]. In the limiting case, when $\kappa \rightarrow \infty$ (e.g $\kappa=10$ ), the distribution function approaches the familiar Maxwellian form (Figure 1). Low values of $\kappa$ represent distributions with relatively large component of particles with speeds greater than the thermal speed ("superthermal particles") and an associated reduction in "thermal" particles, as one observes in a "hard" spectrum [9].

Apart from kappa distributed electrons, electron populations can suit other non-thermal distributions eg. Cairn's distribution [10]. Recently, [5], used Cairn's distributed electrons in an e-p-i plasma and [11] applied the distribution on a 4-component complex dusty plasma. In both cases, solitons of both polarities were found to exist. However, for a $\kappa$ distributed electron population, 


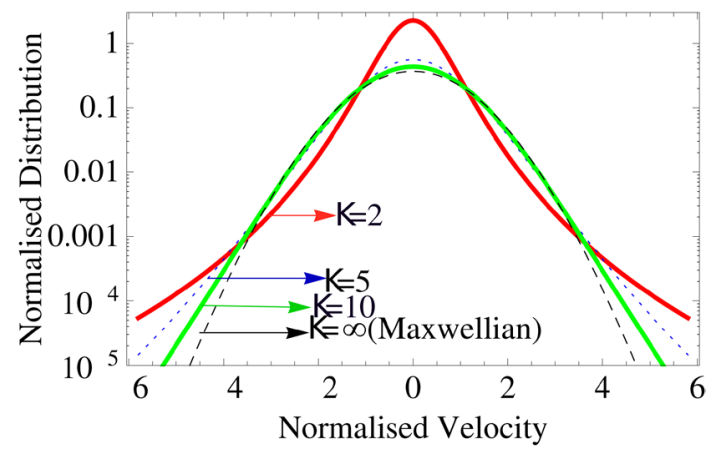

Figure 1. Comparision of generalised Lorentzian distribution for the spectral index $\kappa=2,5,10$, and $\kappa=$ $\infty$ (corresponding to Maxwellian distribution).

solitons of both polarities may not arise. Therefore, this study investigated the effect of using $\kappa$ distributed electrons in an e-p-i plasma.

This paper is therefore structured with the following sections: A brief introduction, description of the model, discussion of results and finally we present a conclusion.

\section{Description of Plasma Model}

We considered a simple three species unmagnetised and collisionless plasma model whose electrons are kappa-distributed. The other species are warm inertial adiabatic fluid ions and Boltzmann-distributed positrons, thereby referred to as an e-p-i plasma. The electrons, positrons, and ions are considered to have temperatures $T_{e}, T_{p}$ and $T_{i}$ respectively. Again, the electrons, ions, and positrons are assumed have densities of $N_{e}, N_{p}$ and $N_{i}$ respectively.

The charge equilibrium equation for the system when electrostatic potential, $\varphi=0$ is given by

$$
f_{p}=1-f
$$

where $f=\frac{N_{i 0}}{N_{e 0}}$ is the ratio of equilibrium number density of ions to electrons, $f_{p}=\frac{N_{p 0}}{N_{e 0}}$ is the ratio of equilibrium number density of positrons to electrons.

Integrating the adopted kappa distribution Equation (1), the velocity space gives the unnormalised electron number density as

$$
N_{e}(\varphi)=\int_{-\infty}^{+\infty} F_{\kappa}(v) \mathrm{d} v=N_{e 0}\left[1-\frac{e \varphi}{\left(\kappa-\frac{3}{2}\right) K_{B} T_{e}}\right]^{-\left(\kappa-\frac{1}{2}\right)} .
$$

It can be noted that Equation (3) is only valid for $\kappa>\frac{3}{2}$, and kappa distributions reduce to Maxiwellian distributions, when $\kappa \rightarrow \infty$ [12], while for low values of $\kappa$, "hard" spectrum with strong non-Maxiwellian tail following a power-law at high speeds are obtained [7]. Normalizing by appropriate scaling 
quantities, the electron number density is obtained in dimensionless form as

$$
n_{e}(\phi)=\left(1-\frac{\phi}{\kappa-\frac{3}{2}}\right)^{-\left(\kappa-\frac{1}{2}\right)}
$$

where $n_{e}$ is particle density and the electrostatic potential $\phi$ are scaled as $\phi=\frac{e \varphi}{K_{B} T_{e}}$ and $n_{e}=\frac{N_{e}}{N_{e 0}}$.

The positrons follow the Boltzmann distribution given as,

$$
N_{p}(\varphi)=N_{p 0} \exp \left[-\frac{e \varphi}{K_{B} T_{p}}\right] \text {. }
$$

Normalising with respect to $N_{e 0}$, the number density for positrons may be written in a dimensionless form as

$$
n_{p}(\phi)=f_{p} \exp [-\tau \phi]
$$

where, $\tau=\frac{T_{e}}{T_{p}}, n_{p}=\frac{N_{p}}{N_{e 0}}$ and $f_{p}=\frac{N_{p 0}}{N_{e 0}}$.

For ions, the density is obtained from the fluid equations. These are continuity, pressure, and momentum equations written respectively as follows:

$$
\begin{gathered}
\frac{\partial N_{i}}{\partial t^{\prime}}+\frac{\partial}{\partial X^{\prime}}\left(N_{i} V_{i}\right)=0, \\
\frac{\partial P_{i}}{\partial t^{\prime}}+V_{i} \frac{\partial P_{i}}{\partial X^{\prime}}+3 P_{i} \frac{\partial V_{i}}{\partial X^{\prime}}=0, \\
\frac{\partial V_{i}}{\partial t^{\prime}}+V_{i} \frac{\partial V_{i}}{\partial X^{\prime}}+\frac{q_{i}}{m_{i}} \frac{\partial \varphi}{\partial X^{\prime}}+\frac{1}{N_{i} m_{i}} \frac{\partial P_{i}}{\partial X^{\prime}}=0,
\end{gathered}
$$

where, $V$ and $P_{i}$ are the un-normalized ion fluid speed and ion thermal pressure respectively; $m_{i}\left(q_{i}\right)$ is the ion mass (charge), and $X^{\prime}\left(t^{\prime}\right)$ is the un-normalized space (time) variable.

This system of Equations (7)-(9) are normalized by introducing dimensionless quantities $\left(x, t, n_{i}, u_{i}, p_{i}\right.$ and $\left.\phi\right)$ such that; $\frac{N_{i}}{N_{i 0}}=n_{i} ; \frac{X^{\prime}}{\lambda_{\text {Deff }}}=x ; \frac{V_{i}}{C_{s}}=u_{i}$; $t^{\prime}\left(\omega_{p i}\right)=t ; \phi=\left(e \varphi / K_{B} T_{e}\right)$ and $\frac{P_{i}}{n_{0} K_{B} T_{i}}=p_{i}$. Here, $\lambda_{\text {Deff }}=\left(\frac{\varepsilon_{o} K_{B} T_{e}}{N_{i 0} e^{2}}\right)^{\frac{1}{2}}$ is Debye length, $C_{s}=\left(\frac{K_{B} T_{e}}{m_{i}}\right)^{\frac{1}{2}}$ is ion acoustic speed, while $\omega_{p i}=\left(\frac{N_{i 0} e^{2}}{\varepsilon_{o} m_{i}}\right)^{\frac{1}{2}}$ is plasma frequency with $m_{i}$ and $e$ the ion mass and electronic charge respectively.

The normalised equations are obtained as:

$$
\begin{gathered}
\frac{\partial n_{i}}{\partial t}+\frac{\partial}{\partial x}\left(n_{i} u_{i}\right)=0 \\
\frac{\partial p_{i}}{\partial t}+u_{i} \frac{\partial p_{i}}{\partial x}+3 p_{i} \frac{\partial u_{i}}{\partial x}=0
\end{gathered}
$$




$$
\frac{\partial u_{i}}{\partial t}+u_{i} \frac{\partial u_{i}}{\partial x}+\frac{\partial \phi}{\partial x}+\frac{\sigma_{i}}{n_{i}} \frac{\partial p_{i}}{\partial x}=0
$$

In transforming to the stationary frame, the solution of nonlinear equations of a wave depends on $x$ and $t$ through the variable $\xi=x-M t$. Here $M$ is the Mach number (normalized by the phase speed of the ion-acoustic waves). Thus, $\frac{\partial}{\partial \xi}=-M \frac{\partial}{\partial t}$ and $\frac{\partial}{\partial \xi}=\frac{\partial}{\partial x}$. We can now integrate Equations (10)-(12) applying boundary conditions as $|\xi| \rightarrow \infty ; u_{i}, p_{i}$ and $\phi \rightarrow 0$, and eliminating $u_{i}$ between the three expressions to obtain the ion density equation as,

$$
n_{i}=\frac{1}{2 \sqrt{3 \sigma_{i}}}\left\{\left[A^{+}-2 \phi\right]^{\frac{1}{2}} \pm\left[A^{-}-2 \phi\right]^{\frac{1}{2}}\right\},
$$

where $A^{+}=M+\left(\sqrt{3 \sigma_{i}}\right)^{2}$ and $A^{-}=M-\left(\sqrt{3 \sigma_{i}}\right)^{2}$.

It is easy to show from Equation (13) that for $M>\left(\sqrt{3 \sigma_{i}}\right)^{2}$, the negative sign is used and for $M<\left(\sqrt{3 \sigma_{i}}\right)^{2}$, the positive is used. The negative sign corresponds to supersonic species [13]. The negative sign satisfies the boundary condition of $n_{i} \rightarrow 1$ with $\phi \rightarrow 0$ as $\xi \rightarrow \pm \infty$. As a result, the ion density is obtained as

$$
n_{i}=\frac{1}{2 \sqrt{3 \sigma_{i}}}\left\{\left[A^{+}-2 \phi\right]^{\frac{1}{2}}-\left[A^{-}-2 \phi\right]^{\frac{1}{2}}\right\} .
$$

Equation (4), (6) and (14) are coupled by Poisson's equation,

$$
\varepsilon_{0} \frac{\partial^{2} \varphi}{\partial X^{\prime 2}}=\sum_{j=e, p, i} q_{j} N_{j},
$$

where $\varepsilon_{0}$ is permittivity of free space and $q_{i}=e$ (for ions), $q_{p}=e$ (for positrons) and $q_{e}=-e$ (for electrons). Normalising with $N_{e 0}$, gives

$$
\frac{\mathrm{d}^{2} \phi}{\mathrm{d} x^{2}}=n_{e}(\phi)-n_{i}(\phi)-n_{p}(\phi) .
$$

In the stationary frame,

$$
\frac{\mathrm{d}^{2} \phi}{\mathrm{d} \xi^{2}}=n_{e}(\phi)-n_{i}(\phi)-n_{p}(\phi)=G(\phi, M) .
$$

Therefore,

$$
\frac{1}{2} \frac{\mathrm{d}}{\mathrm{d} \phi}\left(\frac{\mathrm{d} \phi}{\mathrm{d} \xi}\right)^{2}=\frac{\mathrm{d}^{2} \phi}{\mathrm{d} \xi^{2}}=n_{e}(\phi)-n_{i}(\phi)-n_{p}(\phi)=G(\phi, M) .
$$

Substituting Equations (4), (6) and (14) into Equation (16), and integrating with respect to $\phi$, the usual energy equation [14] is obtained as

$$
\frac{1}{2}\left(\frac{\mathrm{d} \phi}{\mathrm{d} \xi}\right)^{2}+S(\phi, M)=0
$$

where $S(\phi, M)$ represents a potential well with $\phi$ playing the role of "coordinate" (pseudoposition) and $\xi$ represents the role of "time". From Equation (18) and Equation (19) the Sagdeev potential or pseudopotential is obtained as 


$$
\begin{aligned}
S(\phi, M)= & \sigma_{i}+M^{2}+\frac{1}{6 \sqrt{3 \sigma_{i}}}\left\{\left[A^{-}-2 \phi\right]^{\frac{3}{2}}-\left[A^{+}-2 \phi\right]^{\frac{3}{2}}\right\} \\
& +\frac{f_{p}}{\tau}\left[1-\mathrm{e}^{-\tau \phi}\right]+\left[1-\left(1-\frac{\phi}{\kappa-\frac{3}{2}}\right)^{-\left(\kappa-\frac{3}{2}\right)}\right]
\end{aligned}
$$

\section{Results}

\subsection{Theoretical Analysis}

The derived Sagdeev potential, $S(\phi, M)$ is vital in finding the existence domains of IAS waves. For solitary waves to propagate in an e-p-i plasma, Sagdeev potential, $S(\phi, M)$ and its derivatives with respect to $\phi$ must satisfy the following conditions [15].

1) $S(\phi=0, M)=0=S^{\prime}(\phi=0, M)=0$ at the origin $(\phi=0)$.

2) $S^{\prime \prime}(\phi=0, M)<0$, such that the origin is unstable i.e., $(\phi)$ has maximum at origin.

3) $S\left(\phi_{m}, M\right)=0$ and $S(\phi)<0$ for $0<|\phi|<\left|\phi_{m}\right|$. i.e., $\phi_{m}$ for positive root (positive potential) and $\phi_{m}$ for negative root (negative potential). The primes represent derivatives of $S(\phi, M)$ with respect to $\phi$.

Clearly, from Equation (20), $S(\phi, M)$ and its derivative with respect to $\phi$ vanish at $\phi=0$, hence condition (1) is satisfied. The origin at $\phi=0$ thus defines the equilibrium state, which represents a local maximum of $S(\phi, M)$.

The requirement, $S^{\prime \prime}\left(\phi=0, M_{s}\right)=0$, yields

$$
\frac{1}{2 \sqrt{3 \sigma_{i}}}\left[\frac{1}{M_{s}-\sqrt{3 \sigma_{i}}}-\frac{1}{M_{s}+\sqrt{3 \sigma_{i}}}\right]-\tau f_{p}-\left(\frac{\kappa-\frac{1}{2}}{\kappa-\frac{3}{2}}\right)=0,
$$

where $M_{s}$ is the lower Mach number limit below which no soliton can exist. For $S^{\prime \prime}\left(\phi=0, M_{s}\right)<0$, Equation (21) becomes

$$
\frac{1}{2 \sqrt{3 \sigma_{i}}}\left[\frac{1}{M_{s}-\sqrt{3 \sigma_{i}}}-\frac{1}{M_{s}+\sqrt{3 \sigma_{i}}}\right]-\tau f_{p}-\left(\frac{\kappa-\frac{1}{2}}{\kappa-\frac{3}{2}}\right)<0,
$$

which inequality is part of the soliton (existence) condition to be fulfilled as

$$
M_{s}>\left[3 \sigma_{i}+\frac{1}{\tau(1-f)}+\left(\frac{2 \kappa-1}{2 \kappa-3}\right)\right]^{\frac{1}{2}},
$$

i.e., using the charge neutrality condition in Equation (2). For the fixed value of $\kappa$, solitons may exist only for values of the Mach number satisfying

$$
M>M_{s} \text {. }
$$

This is on the basis of investigations by [13] [15] who showed that the existence of solitons required $M>M_{s}$. This implies that solitons can have finite amplitudes at $M_{s}$ in the region where solitons with both polarity exit. For 
$\kappa \rightarrow \infty, \sigma_{i}=0$ and $\tau=1$, Equation (23) reduces to the familiar expression

$$
M_{s}^{2}>\frac{1}{1-f} \text {. }
$$

It follows that if Equation (24) is the "true" Mach number, the structures would be truly supersonic.

Figure 2(a) shows the necessary minimum Mach number for the existence domains of solitary structures. For the three component plasma, Figure 2(a), shows that there are two acoustic existence ranges for the solitary waves i.e., between $M_{s}=0.0221485$, before the thermal velocity and $M_{s}=0.510656$ after the thermal velocity. Therefore, $M_{s}=0.510656$ is the minimum Mach number limit below which no solitary waves exist for this current model. For the case of this study, solitons with positive polarity are limited by infinite compression of the inertial ions. This is achieved when $\phi \rightarrow \phi_{i 1}=\frac{1}{2}\left(M-\sqrt{3 \sigma_{i}}\right)^{2}$, leading to a sufficient condition, $S\left(\phi_{i 1}, M\right)>0$. After finding the minimum Mach number, $M_{s}$, for the nonlinear solitary structures, the maximum Mach number, $M_{u}$ was found using Equation (14). Another limiting potential was obtained to be $\phi_{i 2}=\frac{1}{2}\left(M+\sqrt{3 \sigma_{i}}\right)^{2}$.

Both $\phi>\phi_{i 1}$ and $\phi>\phi_{i 2}$ yields a complex $n_{i}$. Therefore, $\phi>\phi_{i 1}$ and $\phi>\phi_{i 2}$ are the practical constraints limiting positive potential solitons, under the condition $S\left(\phi \geq \phi_{i 1}\right)>0$ and $S\left(\phi=\phi_{i 2}\right)>0$. The critical limiting potential $\phi_{\max }=\phi_{i 2}$, since $\phi_{i 2}<\phi_{i 1}$ for all $M$ and $f$. Therefore, the requirement $S\left(\phi_{\max }, M_{u}\right)=0$ leads to the upper limit on $M$ as $M_{u}=0.631122$ for the existence of positive solitons (see Figure 2(b)). In this case of a three component plasma model, the solution for the existence domains exist in the range $0.510656<M_{u}<0.631122$.

\subsection{Numerical Analysis}

Numerical solutions of the existence domains supported by the plasma model

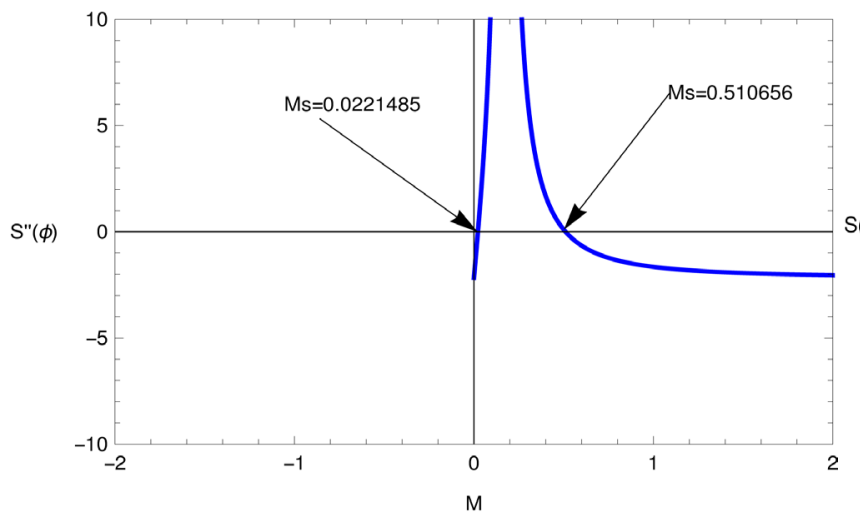

(a)

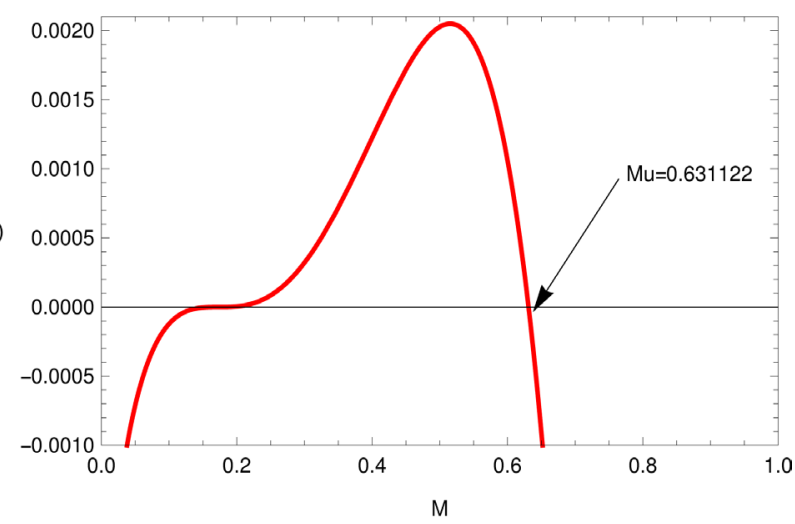

(b)

Figure 2. (a) A graph showing $S^{\prime \prime}(\phi)$ against $M$ for the existence domains in an e-p-i plasma. (b) A graph showing $S(\phi)$ against $M$ for the existence domains in a three component plasma. 
consisting of three species were obtained using the Sagdeev potential in Equation (20).

\subsubsection{Existence Diagrams}

Figure 3(a) shows the existence domains for solitons in parameter space of maximum and minimum Mach numbers for a plasma with electrons having the same temperature as positrons, i.e., $\tau=\frac{T_{e}}{T_{p}}=1$. Positive solitons at different values of $\kappa$ (i.e., $\kappa=3$ are indicated by blue dots, $\kappa=7$, by red thick line, and $\kappa=100$ by black dashed line where $\sigma_{i}=\frac{T_{i}}{T_{e}}=\frac{1}{100}$ and $f_{p}=0.1$. It can clearly be seen that the existence domains of finite amplitude ion acoustic solitary waves are confined within the limits of Mach number given by Equation (23) and (24), respectively. For the values of $\kappa$ used, the positive potential solitons exist for the full range $\left(0<f_{p}<1\right)$ of the density ratio. For this case, the existence domain increased with increase in $\kappa$.

Figure 3(b) shows numbers, $M$ plotted against the electron-positron temperature ratio, $\tau$ for various values of ion-to-electron temperature ratio ( $\sigma_{i}=0.01$ plotted in blue dots, $\sigma_{i}=0.03$ in red thick line, and $\sigma_{i}=0.05$ in black dashes) where $\kappa=3, \tau=1$ and $f_{p}=0.5$. Finally, it was observed that increasing the electron-positron (e-p) temperature ratio, $\tau$ increased the Mach number of the solitary wave. It is predictable that when temperature ratio, $\sigma_{i}$ is increased, a corresponding increase in the existence domains of the finite amplitude ion acoustic solitary waves is obtained. Having discused the existence domains of IAS waves in an e-p-i plasma, the effects of kappa distributed electrons and inertial warm ions on propagation of IAS waves is discussed next.

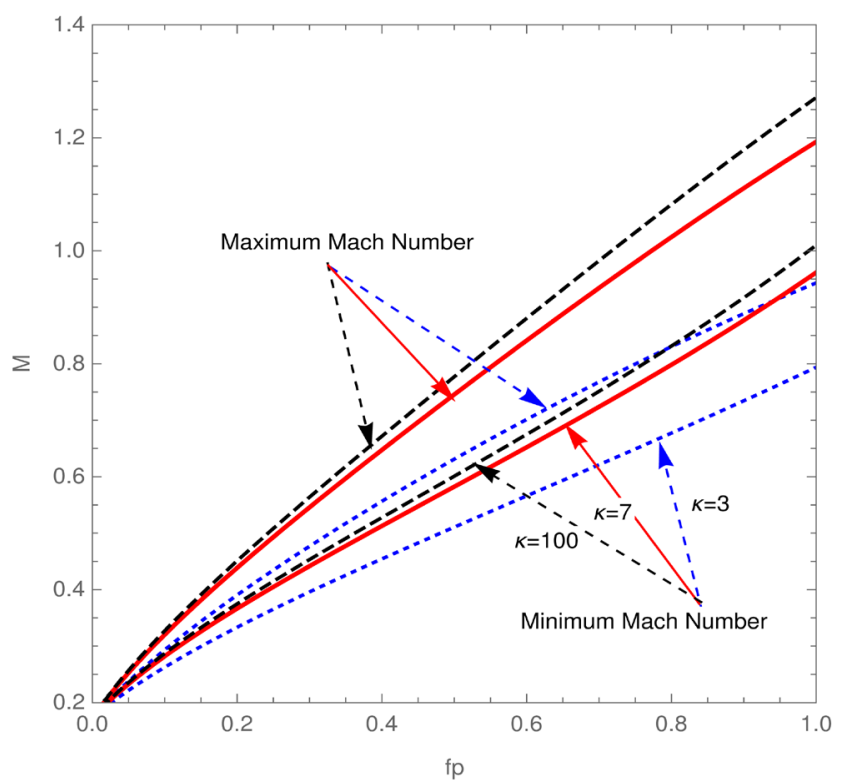

(a)

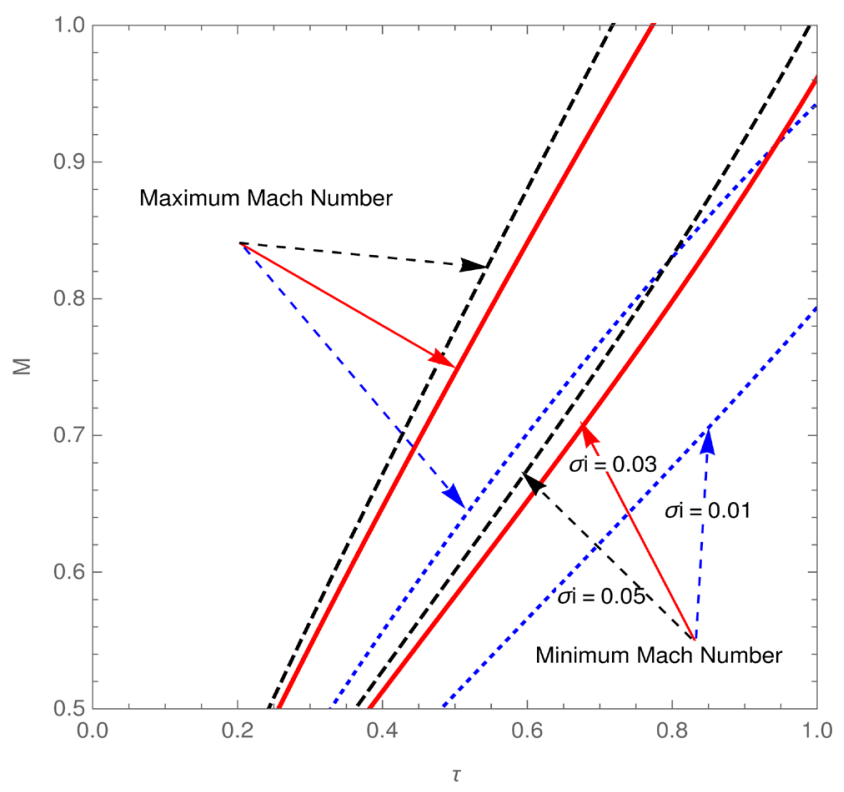

(b)

Figure 3. (a) The Mach numbers, $M$ against the positron-to-electon density ratio $f_{p}$ for different values of non-thermal parameter $\kappa$. (b) The Mach numbers, $M$ versus the electron-to-positron temperature ratio, $\tau$ for various values of $\sigma_{i}$. 


\subsubsection{Effect of Kappa Distributed Electrons}

Figure 4(a) shows the variation of the Sagdeev potential, $S(\phi)$ with $\phi$ for a fixed Mach number, $M=0.62$, and various values of $\kappa$ ranging from 3 (in purple dots) to 10 (red thick line). Different values of $\kappa$ were used to examine the one that would give a better fit to the kappa distribution. It is clearly observed that for a lower value of $\kappa$, the amplitude of the solitary electrostatic potential structures increased from 0.04 for $\kappa=10$, to 0.10 for $\kappa=3$. Hence, for a fixed $M$, a decrease in $\kappa$ causes an increase in the true sound speed. Thus, it follows that a decrease in $\kappa$ leads to larger solitons, as shown in Figure 4(a). Furthermore, the depth of the Sagdeev potential increased from 0.0001 to 0.0015 over the range $(3<\kappa<10)$. Thus, the soliton profile increased as the nonthermal component was increased. When the amplitude increased, it resulted into the soliton profile, which become a bit steeper. Figure 4 (b) is a plot of $\phi(\xi)$ against $\xi$ showing the bell shaped electrostatic potential. It is clearly seen that for lower values of $\kappa$, say $\kappa=3$, the potential amplitude increased as the width decreased, while for high values $(\kappa=5)$, the potential amplitude decreased as the width of the solitary structures increased.

\subsubsection{Effect of Inertial Warm Ions}

Figure 5(a) showed the variation of Sagdeev potential, $S(\phi)$ with $\phi$ for a fixed $M(0.60), \kappa=3$, and various values of $\sigma_{i}$, ranging from $\sigma_{i}=0.015$ (thick) to $\sigma_{i}=0.025$ (black thick line) of solitary electrostatics potential structure. When the different values of ion-to-electron temperature rations, $\sigma_{i}$ were reduced from 0.025 to 0.015 and the potential increased from 0.0378 to 0.06436, particle trapping was exhibited, which implied the formation of solitons. Thus, for fixed $M$ and $\kappa$, a decrease in $\sigma_{i}$ resulted into high amplitude solitons. Again, Figure 5(a) showed that the depth of Sagdeev potential increased from 0.00005786 to 0.000265 . This implied that solitons exists with potential wells of $\phi(\xi)$ profiles at different depths. In non-thermal

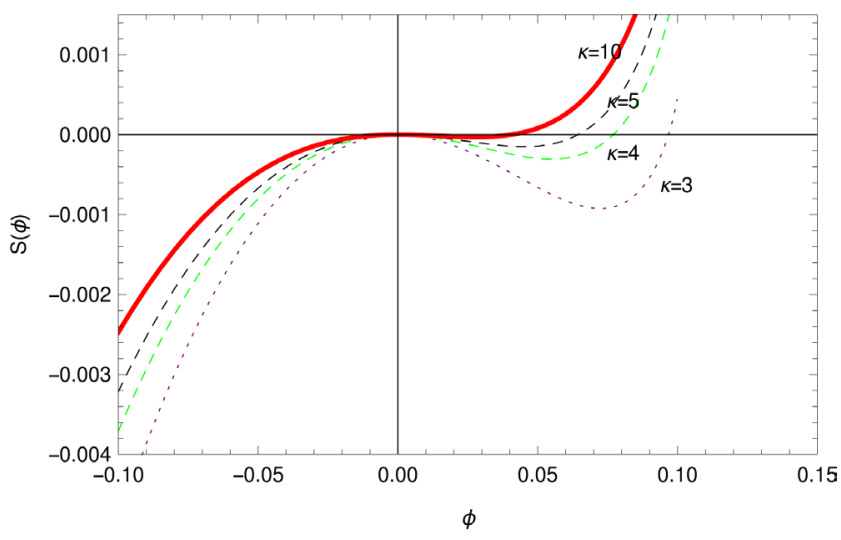

(a)

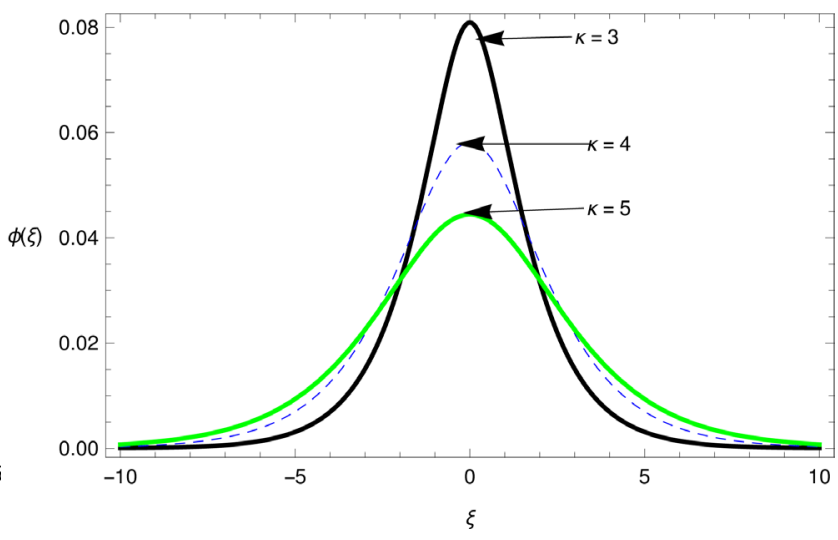

(b)

Figure 4. (a) Variation of $S(\phi)$ against $\phi$ for $M=0.62, f_{p}=0.5, \tau=1, \sigma_{i}=0.01$ and various values of kappa: $\kappa=3$ (purple dots), $\kappa=4$ (green dashes), $\kappa=5$ (black dots), $\kappa=10$ (red thick line). (b) Arbitrary amplitude ion acoustic pulses, $\phi(\xi)$ against $\xi$ for different values of $\kappa: M=0.60, f_{p}=0.5, \sigma_{i}=0.01, \tau=1$, and $\kappa=3$ (black thick line), $\kappa=4$ (blue dots), and $\kappa=5$ (green thick line). 


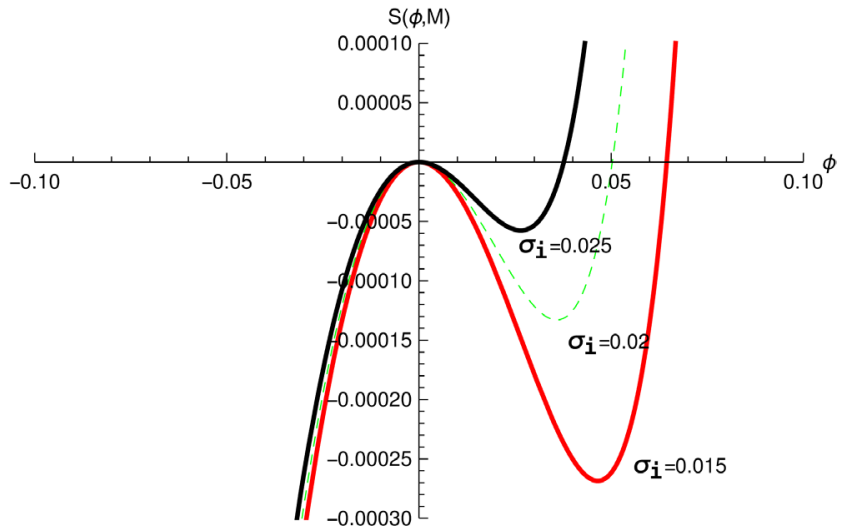

(a)

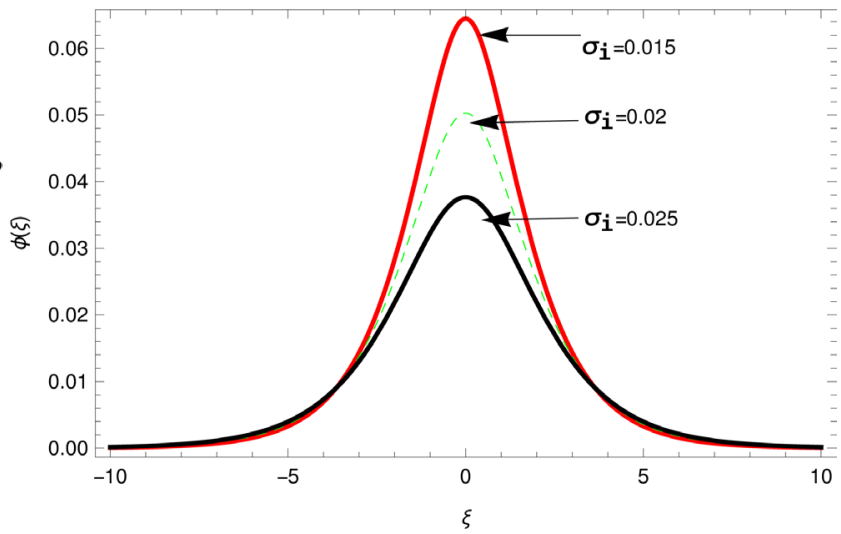

(b)

Figure 5. (a) Variation of $S(\phi)$ against $\phi$ for $M=0.60, f_{p}=0.5, \kappa=3, \tau=1$ and various values of $\sigma_{i}: \sigma_{i}=0.015$ (red thick curve), $\sigma_{i}=0.020$ (green dotted curve), and $\sigma_{i}=0.025$ (black thick curve). (b) Arbitrary amplitude of ion acoustic pulses, $\phi(\xi)$ against $\xi$ for various values of ion-to-electron temperature ratio $\sigma_{i}: M=0.60, f_{p}=0.5, \kappa=3, \tau=1$, and $\sigma_{i}=0.015$ (red thick line), $\sigma_{i}=0.020$ (green dots), and $\sigma_{i}=0.025$ (black thick line).

environment, plasma formation is more likely and amplitude of the soliton is maximum because of greater depth.

In Figure $5(\mathrm{~b})$, the electrostatic potential, $\phi(\xi)$ is plotted against $\xi$, for varying $\sigma_{i}$ values. Only bell shaped electrostatic potentials are obtained. The potential amplitude increased as ion-to-electron temperature ratio, $\sigma_{i}$ decreased. However, the width of the solitary structures increased with increasing ion-toelectron temperature ratio, $\sigma_{i}$. Furthermore, it is shown that the potential amplitude $\phi(\xi)$, in the non-thermal case was higher for smaller value of $\sigma_{i}=0.015$, but this trend became weaker for bigger values, say $\sigma_{i}=0.025$. It was noted that the effect of $\sigma_{i}$ on the amplitude of the solitary waves was weaker for non-thermal case when $\sigma_{i}>0.020$ as shown by the black thick curve and green dotted curve in Figure 5(b). In conclusion, the effects of ion temperature ratio $\sigma_{i}$ on the properties of the new solitary wave are more pronounced for smaller $\sigma_{i}$.

\section{Conclusion}

The study findings show a fully non-linear ion acoustic solitary wave in e-p-i plasma with three species (i.e., warm inertial adiabatic ion fluid, kappadistributed electrons, and Boltzmann-distributed positrons). The existence of large amplitude ion acoustic solitons of positive electrostatic potential was obtained. Numerical solution of the energy integral equation showed that positive solitary waves existed with a property of having faster pulses with taller and slender amplitudes. Furthermore, increasing the non-thermal parameter $\kappa$, it was found that the amplitudes of ion acoustic solitons were reduced when a significant fraction of positrons is present in the plasma. This implied that the positron component hindered the propagation speed of the solitons. These results are capable of enhancing the understanding of non-linear structures in e-p-i plasmas found in certain astronomical environments containing non- 
thermal electrons as a component.

\section{Acknowledgements}

We acknowledge the funding from East African Astronomical Research Network (EAARN) and support from the International Science Program (ISP) based in Uppsala University in Sweden.

\section{References}

[1] Shukla, P.K and Mamun, A.A. (2002) Introduction to Dusty Plasma Physics. Institute of Physics, Bristol, 1-2.

[2] Popel, S.I., Vladimirov, S.V and Shukla, P.K. (1995) Physics of Plasmas, 2, 716. https://doi.org/10.1063/1.871422

[3] Nejoh, Y.N. (1997) Australian Journal Physics, 50, 309-317. https://doi.org/10.1071/P96064

[4] Moslem, W.M., Kourakis, I., Shukla, P.K and R. Schlickeiser, R. (2007) Physics of Plasmas, 14, Article ID: 102901. https://doi.org/10.1063/1.2795127

[5] Baluku, T.K. and Hellberg, M.A. (2011) Plasma Physics and Controlled Fusion, 53, Article ID: 095007. https://doi.org/10.1088/0741-3335/53/9/095007

[6] Xue, S., Thorne, R.M and Summers, D. (1993) Journal of Geophysical Research, 98, 17475-17484. https://doi.org/10.1029/93JA00790

[7] Summers, D. and Thorne, R.M. (1991) Physics of Plasma, 3, 1835. https://doi.org/10.1063/1.859653

[8] Vasyliunas, V.M. (1968) Journal of Geophysical Research, 73, 2839.

[9] Saini, N.S., Kourakis, I and Hellberg, M.A. (2009) Physics of Plasmas, 16, Article ID: 062903. https://doi.org/10.1063/1.3143036

[10] Cairns, R.A., Mamun, A.A., Bingham, R., Dendy, R.O., Nairn, C.M.C. and Shukla, P.K. (1995) Geophysical Research Letters, 22, 2709-2712. https://doi.org/10.1029/95GL02781

[11] Habumugisha, I., Anguma, S.K., Jurua, E. and Noreen, N. (2016) International Journal of Astronomy and Astrophysics, 6, 1-7. https://doi.org/10.4236/ijaa.2016.61001

[12] Baluku, T.K. Hellberg, M.A. Kourakis, I. and Saini, N.S (2010) Physics of Plasmas, 17, Article ID: 053702. https://doi.org/10.1063/1.3400229

[13] Verheest, F. and Pillay, S.R. (2008) Physics of Plasmas, 15, Article ID: 013703. https://doi.org/10.1063/1.2831025

[14] Verheest, F. and Hellberg, M.A. and Saini, N.S. and Kourakis, I. (2011) Physics of Plasmas, 18, Article ID: 042309.

[15] Baluku, T.K. and Hellberg, M.A. (2008) Physics of Plasmas, 15, Article ID: 123705. https://doi.org/10.1063/1.3042215 
Submit or recommend next manuscript to SCIRP and we will provide best service for you:

Accepting pre-submission inquiries through Email, Facebook, LinkedIn, Twitter, etc. A wide selection of journals (inclusive of 9 subjects, more than 200 journals)

Providing 24-hour high-quality service

User-friendly online submission system

Fair and swift peer-review system

Efficient typesetting and proofreading procedure

Display of the result of downloads and visits, as well as the number of cited articles Maximum dissemination of your research work

Submit your manuscript at: http://papersubmission.scirp.org/

Or contact jmp@scirp.org 